\title{
Posterior Probability on Finite Set ${ }^{1}$
}

\author{
Hiroyuki Okazaki \\ Shinshu University \\ Nagano, Japan
}

\begin{abstract}
Summary. In [14] we formalized probability and probability distribution on a finite sample space. In this article first we propose a formalization of the class of finite sample spaces whose element's probability distributions are equivalent with each other. Next, we formalize the probability measure of the class of sample spaces we have formalized above. Finally, we formalize the sampling and posterior probability.
\end{abstract}

MML identifier: DIST_2, version: $\underline{8.0 .015 .3 .1162}$

The notation and terminology used in this paper have been introduced in the following papers: [11], [1], [14], [17], [3], [5], [20], [10], [6], [7], [4], [19], [22], [25], [18], [2], [8], [13], [15], [12], [23], [24], [16], [21], and [9].

\section{Equivalent Distributed Finite and Distributed Sample Spaces}

The following propositions are true:

(1) Let $Y$ be a non empty finite set and $s$ be a finite sequence of elements of $Y$. If $Y=\{1\}$ and $s=\langle 1\rangle$, then FDprobSEQ $s=\langle 1\rangle$.

(2) Let $S$ be a non empty finite set, $p$ be a probability distribution finite sequence on $S$, and $s$ be a finite sequence of elements of $S$. If FDprobSEQ $s=p$, then distribution $(p, S)=$ the equivalence class of $s$ and $s \in \operatorname{distribution}(p, S)$.

(3) Let $S$ be a non empty finite set and $x$ be an element of $S$. Then $x \in \operatorname{rng} \operatorname{CFS}(S)$ and there exists a natural number $n$ such that $n \in$ $\operatorname{dom} \operatorname{CFS}(S)$ and $x=(\operatorname{CFS}(S))(n)$ and $n \in \operatorname{Seg} \overline{\bar{S}}$.

\footnotetext{
${ }^{1}$ This work is supported by JSPS KAKENHI 21240001.
} 
Let $S$ be a non empty finite set. One can check that every non empty finite set is non empty.

Let $S$ be a non empty finite set and let $D$ be an element of the distribution family of $S$. We see that the element of $D$ is a finite sequence of elements of $S$.

One can prove the following proposition

(4) Let $S$ be a non empty finite set, $D$ be an element of the distribution family of $S$, and $s, t$ be elements of $D$. Then $s$ and $t$ are probability equivalent.

Let $S$ be a non empty finite set and let $D$ be an element of the distribution family of $S$. We introduce $D$ is well distributed as a synonym of $D$ has non empty elements.

We now state the proposition

(5) Let $S$ be a non empty finite set and $s$ be a finite sequence of elements of $S$. Then for every set $x$ holds $\operatorname{Prob}_{\mathrm{D}}(x, s)=0$ if and only if $s$ is empty.

Let $S$ be a non empty finite set. Observe that every non empty finite set which is well distributed

We now state the proposition

(6) Let $S$ be a non empty finite set and $D$ be an element of the distribution family of $S$. Then $D$ is not well distributed if and only if $D=\left\{\varepsilon_{S}\right\}$.

Let $S$ be a non empty finite set. An equivalent distributed sample spaces family of $S$ is a well distributed element of the distribution family of $S$.

Let $S$ be a non empty finite set. One can verify that the uniform distribution $S$ is well distributed.

One can prove the following proposition

(7) Let $S$ be a non empty finite set and $D$ be an equivalent distributed sample spaces family of $S$. Then (GenProbSEQ $S)(D)$ is a probability distribution finite sequence on $S$.

\section{Probability Measure of Equivalent Distributed Finite and Distributed Sample Spaces}

Let $S$ be a non empty finite set and let $a$ be an element of $S$. The functor $|\bullet: a|_{\mathbb{N}}$ yielding an element of $\mathbb{N}$ is defined by:

(Def. 1) $|\bullet: a|_{\mathbb{N}}=a \leftrightarrow \operatorname{CFS}(S)$.

Let $S$ be a non empty finite set and let $D$ be an equivalent distributed sample spaces family of $S$. The probability finite sequence of $D$ yields a probability distribution finite sequence on $S$ and is defined by:

(Def. 2) The probability finite sequence of $D=(\operatorname{GenProbSEQ} S)(D)$.

Let $j_{1}$ be a Boolean-valued function. The true event of $j_{1}$ yielding an event of $\operatorname{dom} j_{1}$ is defined as follows: 
(Def. 3) The true event of $j_{1}=j_{1}{ }^{-1}(\{$ true $\})$.

The following proposition is true

(8) Let $S$ be a non empty finite set, $f$ be an $S$-valued function, and $j_{1}$ be a function from $S$ into Boolean. Then the true event of $j_{1} \cdot f$ is an event of $\operatorname{dom} f$.

Let $S$ be a non empty finite set, let $D$ be an equivalent distributed sample spaces family of $S$, let $s$ be an element of $D$, and let $j_{1}$ be a function from $S$ into Boolean. The functor $\operatorname{Prob}\left(j_{1}, s\right)$ yielding a real number is defined as follows:

(Def. 4) $\operatorname{Prob}\left(j_{1}, s\right)=\frac{\overline{\overline{\text { the true event of } j_{1} \cdot s}}}{\text { len } s}$.

The following propositions are true:

(9) Let $S$ be a non empty finite set, $D$ be an equivalent distributed sample spaces family of $S, s$ be an element of $D, j_{1}$ be a function from $S$ into Boolean, $F$ be a non empty finite set, and $E$ be an event of $F$. If $F=\operatorname{dom} s$ and $E=$ the true event of $j_{1} \cdot s$, then $\operatorname{Prob}\left(j_{1}, s\right)=\mathrm{P}(E)$.

(10) Let $S$ be a non empty finite set, $D$ be an equivalent distributed sample spaces family of $S, a$ be an element of $S, s$ be an element of $D$, and $j_{1}$ be a function from $S$ into Boolean. If for every set $x$ holds $x=a$ iff $j_{1}(x)=$ true, then $\operatorname{Prob}\left(j_{1}, s\right)=\operatorname{Prob}_{\mathrm{D}}(a, s)$.

Let $S$ be a set, let $s$ be a finite sequence of elements of $S$, and let $A$ be a subset of $\operatorname{dom} s$. The functor $\operatorname{extract}(s, A)$ yielding a finite sequence of elements of $S$ is defined by:

(Def. 5) $\operatorname{extract}(s, A)=s \cdot \operatorname{CFS}(A)$.

We now state several propositions:

(11) Let $S$ be a set, $s$ be a finite sequence of elements of $S$, and $A$ be a subset of $\operatorname{dom} s$. Then len $\operatorname{extract}(s, A)=\overline{\bar{A}}$ and for every natural number $i$ such that $i \in \operatorname{dom} \operatorname{extract}(s, A)$ holds $(\operatorname{extract}(s, A))(i)=s((\operatorname{CFS}(A))(i))$.

(12) Let $S$ be a non empty finite set, $s$ be a finite sequence of elements of $S, A$ be a subset of $\operatorname{dom} s$, and $f$ be a function. If $f=\operatorname{CFS}(A)$, then $\operatorname{extract}(s, A) \cdot f^{-1}=s\lceil A$.

(13) Let $S$ be a non empty finite set, $f$ be an $S$-valued function, $j_{1}$ be a function from $S$ into Boolean, and $n$ be a set. Suppose $n \in \operatorname{dom} f$. Then $n \in$ the true event of $j_{1} \cdot f$ if and only if $f(n) \in$ the true event of $j_{1}$.

(14) Let $S$ be a non empty finite set, $f$ be an $S$-valued function, and $j_{1}$ be a function from $S$ into Boolean. Then the true event of $j_{1} \cdot f=f^{-1}$ (the true event of $j_{1}$ ).

(15) Let $S$ be a non empty finite set, $D$ be an equivalent distributed sample spaces family of $S, s$ be an element of $D$, and $j_{1}$ be a function from $S$ into Boolean. Then there exists a subset $A$ of dom freqSEQ $s$ such that $A=$ the true event of $j_{1} \cdot \operatorname{CFS}(S)$ and $\overline{\overline{\text { the true event of } j_{1} \cdot s}}=$ 
$\sum \operatorname{extract}($ freqSEQ $s, A$ ).

(16) Let $S$ be a non empty finite set, $D$ be an equivalent distributed sample spaces family of $S$, and $s$ be an element of $D$. Then freqSEQ $s=$ len $s$. FDprobSEQ $s$.

(17) Let $S$ be a non empty finite set, $D$ be an equivalent distributed sample spaces family of $S, s, t$ be elements of $D$, and $j_{1}$ be a function from $S$ into Boolean. Then $\operatorname{Prob}\left(j_{1}, s\right)=\operatorname{Prob}\left(j_{1}, t\right)$.

Let $S$ be a non empty finite set, let $D$ be an equivalent distributed sample spaces family of $S$, and let $j_{1}$ be a function from $S$ into Boolean. The functor $\operatorname{Prob}\left(j_{1}, D\right)$ yielding a real number is defined by:

(Def. 6) For every element $s$ of $D$ holds $\operatorname{Prob}\left(j_{1}, D\right)=\operatorname{Prob}\left(j_{1}, s\right)$.

Next we state the proposition

(18) For every non empty finite set $S$ and for every element $s$ of $S^{*}$ and for every function $j_{1}$ from $S$ into Boolean holds $\operatorname{Coim}\left(j_{1} \cdot s\right.$, true $) \in 2^{\operatorname{dom} s}$.

Let $S$ be a set and let $S_{1}$ be a subset of $S$. The membership decision of $S_{1}$ yielding a function from $S$ into Boolean is defined as follows:

(Def. 7) The membership decision of $S_{1}=\chi_{\left(S_{1}\right), S}$.

The following propositions are true:

(19) For every non empty finite set $S$ and for every subset $B_{1}$ of $S$ there exists a function $j_{1}$ from $S$ into Boolean such that $\operatorname{Coim}\left(j_{1}\right.$, true $)=B_{1}$.

(20) Let $S$ be a non empty finite set, $s$ be an element of $S^{*}, f$ be a function

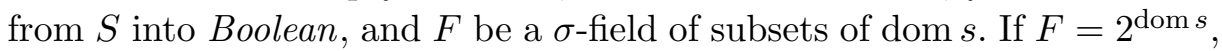
then $\operatorname{Coim}(f \cdot s$, true $)$ is an event of $F$.

(21) Let $S$ be a non empty finite set, $s$ be an element of $S^{*}$, and $f, g$ be functions from $S$ into Boolean. Then $\operatorname{Coim}((f \vee g) \cdot s$, true $)=\operatorname{Coim}(f$. $s$, true $) \cup \operatorname{Coim}(g \cdot s$, true $)$.

(22) Let $S$ be a non empty finite set, $s$ be an element of $S^{*}$, and $f, g$ be functions from $S$ into Boolean. Then $\operatorname{Coim}((f \wedge g) \cdot s$, true $)=\operatorname{Coim}(f$. $s$, true $) \cap \operatorname{Coim}(g \cdot s$, true $)$.

(23) Let $S$ be a non empty finite set, $s$ be an element of $S^{*}$, and $f$ be a function from $S$ into Boolean. Then $\operatorname{Coim}(\neg f \cdot s$, true $)=\operatorname{dom} s \backslash \operatorname{Coim}(f \cdot s$, true $)$.

(24) Let $S$ be a non empty finite set, $D$ be an equivalent distributed sample spaces family of $S, s$ be an element of $D$, and $f, g$ be functions from $S$ into Boolean. Then $\operatorname{Prob}(f \vee g, s)=\frac{\overline{\overline{(\text { the true event of } f \cdot s) \cup(\text { the true event of } g \cdot s)}}}{\operatorname{len} s}$.

(25) Let $S$ be a non empty finite set, $D$ be an equivalent distributed sample spaces family of $S, s$ be an element of $D$, and $f, g$ be functions from $S$ into Boolean. Then $\operatorname{Prob}(f \wedge g, s)=\frac{\overline{\overline{(\text { the true event of } f \cdot s) \cap(\text { the true event of } g \cdot s)}}}{\operatorname{len} s}$.

(26) Let $S$ be a non empty finite set, $D$ be an equivalent distributed sample spaces family of $S, s$ be an element of $D$, and $f$ be a function from $S$ into 
Boolean. Then $\operatorname{Prob}(\neg f, s)=1-\operatorname{Prob}(f, s)$.

(27) Let $S$ be a non empty finite set, $D$ be an equivalent distributed sample spaces family of $S$, and $f, g$ be functions from $S$ into Boolean. Then $\operatorname{Prob}(f \vee g, D)=(\operatorname{Prob}(f, D)+\operatorname{Prob}(g, D))-\operatorname{Prob}(f \wedge g, D)$.

(28) Let $S$ be a non empty finite set, $D$ be an equivalent distributed sample spaces family of $S$, and $f$ be a function from $S$ into Boolean. Then $\operatorname{Prob}(\neg f, D)=1-\operatorname{Prob}(f, D)$.

(29) Let $S$ be a non empty finite set, $D$ be an equivalent distributed sample spaces family of $S$, and $f$ be a function from $S$ into Boolean. If $f=\chi_{S, S}$, then $\operatorname{Prob}(f, D)=1$.

(30) Let $S$ be a non empty finite set, $D$ be an equivalent distributed sample spaces family of $S$, and $f$ be a function from $S$ into Boolean. Then $0 \leq$ $\operatorname{Prob}(f, D)$.

(31) Let $S$ be a non empty finite set, $A, B$ be sets, and $f, g$ be functions from $S$ into Boolean. If $A \subseteq S$ and $B \subseteq S$ and $f=\chi_{A, S}$ and $g=\chi_{B, S}$, then $\chi_{A \cup B, S}=f \vee g$.

(32) Let $S$ be a non empty finite set, $D$ be an equivalent distributed sample spaces family of $S, A, B$ be sets, and $f, g$ be functions from $S$ into Boolean. If $A \subseteq S$ and $B \subseteq S$ and $A$ misses $B$ and $f=\chi_{A, S}$ and $g=\chi_{B, S}$, then $\operatorname{Prob}(f \wedge g, D)=0$.

Let $S$ be a non empty finite set and let $D$ be an equivalent distributed sample spaces family of $S$. A function from Boolean ${ }^{S}$ into $\mathbb{R}$ is said to be a probability on $D$ if:

(Def. 8) For every element $j_{1}$ of Boolean ${ }^{S}$ holds it $\left(j_{1}\right)=\operatorname{Prob}\left(j_{1}, D\right)$.

Let $S$ be a non empty finite set and let $D$ be an equivalent distributed sample spaces family of $S$. The trivial probability of $D$ yields a probability on the trivial $\sigma$-field of $S$ and is defined by the condition (Def. 9).

(Def. 9) Let $x$ be a set. Suppose $x \in$ the trivial $\sigma$-field of $S$. Then there exists a function $c_{1}$ from $S$ into Boolean such that $c_{1}=\chi_{x, S}$ and (the trivial probability of $D)(x)=\operatorname{Prob}\left(c_{1}, D\right)$.

\section{Sampling And Posterior Probability}

Let $S$ be a non empty finite set and let $D$ be an equivalent distributed sample spaces family of $S$. An element of $S$ is called a sample of $D$ if:

(Def. 10) There exists an element $s$ of $D$ such that it $\in \operatorname{rng} s$.

Let $S$ be a non empty finite set, let $D$ be an equivalent distributed sample spaces family of $S$, and let $x$ be a sample of $D$. The functor Prob $x$ yielding a real number is defined as follows:

(Def. 11) $\operatorname{Prob} x=\operatorname{Prob}($ the membership decision of $\{x\}, D)$. 
One can prove the following proposition

(33) Let $S$ be a non empty finite set, $D$ be an equivalent distributed sample spaces family of $S$, and $x$ be a sample of $D$. Then Prob $x=$ (the probability finite sequence of $D)\left(|\bullet: x|_{\mathbb{N}}\right)$.

A non empty subset of $S$ is said to be a sampling RNG of $D$ if:

(Def. 12) There exists a sample $x$ of $D$ such that $x \in$ it.

Let $S$ be a non empty finite set, let $D$ be an equivalent distributed sample spaces family of $S$, and let $X$ be a sampling RNG of $D$. The functor Prob $X$ yielding a real number is defined as follows:

(Def. 13) $\operatorname{Prob} X=\operatorname{Prob}($ the membership decision of $X, D)$.

We now state several propositions:

(34) Let $S$ be a non empty finite set, $X$ be a subset of $S, s, t$ be finite sequences of elements of $S, S_{2}$ be a subset of dom $s$, and $x$ be a subset of $X$. If $S_{2}=s^{-1}(X)$ and $t=\operatorname{extract}\left(s, S_{2}\right)$, then $\overline{\overline{s^{-1}(x)}}=\overline{\overline{t^{-1}(x)}}$.

(35) Let $S$ be a non empty finite set, $X$ be a subset of $S, s, t$ be finite sequences of elements of $S, S_{2}$ be a subset of $\operatorname{dom} s$, and $x$ be a set. If $S_{2}=s^{-1}(X)$ and $t=\operatorname{extract}\left(s, S_{2}\right)$ and $x \in X$, then frequency $(x, s)=\operatorname{frequency}(x, t)$.

(36) Let $S$ be a non empty finite set, $D$ be an element of the distribution family of $S$, and $s$ be a finite sequence of elements of $S$. If $s \in D$, then $D=$ the equivalence class of $s$.

(37) Let $S$ be a non empty finite set, $X$ be a subset of $S$, and $s$ be a finite sequence of elements of $S$. Then $s^{-1}(X)=$ the true event of (the membership decision of $X) \cdot s$.

(38) Let $S$ be a non empty finite set, $X$ be a non empty subset of $S, D$ be an equivalent distributed sample spaces family of $S, s_{1}, s_{2}$ be elements of $D$, $t_{1}, t_{2}$ be finite sequences of elements of $S, S_{3}$ be a subset of dom $s_{1}$, and $S_{4}$ be a subset of dom $s_{2}$. Suppose $S_{3}=s_{1}{ }^{-1}(X)$ and $t_{1}=\operatorname{extract}\left(s_{1}, S_{3}\right)$ and $S_{4}=s_{2}{ }^{-1}(X)$ and $t_{2}=\operatorname{extract}\left(s_{2}, S_{4}\right)$. Then $t_{1}$ and $t_{2}$ are probability equivalent.

The conditional subset of $X$ yields an equivalent distributed sample spaces family of $S$ and is defined by the condition (Def. 14).

(Def. 14) There exists an element $s$ of $D$ and there exists a finite sequence $t$ of elements of $S$ and there exists a subset $S_{2}$ of dom $s$ such that $S_{2}=s^{-1}(X)$ and $t=\operatorname{extract}\left(s, S_{2}\right)$ and $t \in$ the conditional subset of $X$.

Let $f$ be a function from $S$ into Boolean. The functor $\operatorname{Prob}(f, X)$ yielding a real number is defined by:

(Def. 15) $\operatorname{Prob}(f, X)=\operatorname{Prob}(f$, the conditional subset of $X)$.

One can prove the following proposition 
(39) Let $S$ be a non empty finite set, $D$ be an equivalent distributed sample spaces family of $S, X$ be a sampling RNG of $D$, and $f$ be a function from $S$ into Boolean. Then $\operatorname{Prob}(f, X) \cdot \operatorname{Prob} X=\operatorname{Prob}(f \wedge$ the membership decision of $X, D)$.

\section{REFERENCES}

[1] Grzegorz Bancerek. Cardinal numbers. Formalized Mathematics, 1(2):377-382, 1990.

[2] Grzegorz Bancerek. The ordinal numbers. Formalized Mathematics, 1(1):91-96, 1990.

[3] Grzegorz Bancerek and Krzysztof Hryniewiecki. Segments of natural numbers and finite sequences. Formalized Mathematics, 1(1):107-114, 1990.

[4] Czesław Byliński. Basic functions and operations on functions. Formalized Mathematics, 1(1):245-254, 1990.

[5] Czesław Byliński. Finite sequences and tuples of elements of a non-empty sets. Formalized Mathematics, 1(3):529-536, 1990.

[6] Czesław Byliński. Functions and their basic properties. Formalized Mathematics, 1(1):5565, 1990.

[7] Czesław Byliński. Functions from a set to a set. Formalized Mathematics, 1(1):153-164, 1990.

[8] Czesław Byliński. Partial functions. Formalized Mathematics, 1(2):357-367, 1990.

[9] Czesław Byliński. Some basic properties of sets. Formalized Mathematics, 1(1):47-53, 1990.

[10] Agata Darmochwał. Finite sets. Formalized Mathematics, 1(1):165-167, 1990.

[11] Shunichi Kobayashi and Kui Jia. A theory of Boolean valued functions and partitions. Formalized Mathematics, 7(2):249-254, 1998.

[12] Andrzej Kondracki. Basic properties of rational numbers. Formalized Mathematics, 1(5):841-845, 1990.

[13] Andrzej Nędzusiak. $\sigma$-fields and probability. Formalized Mathematics, 1(2):401-407, 1990.

[14] Hiroyuki Okazaki. Probability on finite and discrete set and uniform distribution. Formalized Mathematics, 17(2):173-178, 2009, doi: 10.2478/v10037-009-0020-z.

[15] Hiroyuki Okazaki and Yasunari Shidama. Probability on finite set and real-valued random variables. Formalized Mathematics, 17(2):129-136, 2009, doi: 10.2478/v10037-009-0014-x.

[16] Beata Padlewska. Families of sets. Formalized Mathematics, 1(1):147-152, 1990.

[17] Andrzej Trybulec. Domains and their Cartesian products. Formalized Mathematics, 1(1):115-122, 1990.

[18] Andrzej Trybulec. On the sets inhabited by numbers. Formalized Mathematics, 11(4):341$347,2003$.

[19] Michał J. Trybulec. Integers. Formalized Mathematics, 1(3):501-505, 1990.

[20] Wojciech A. Trybulec. Pigeon hole principle. Formalized Mathematics, 1(3):575-579, 1990.

[21] Zinaida Trybulec. Properties of subsets. Formalized Mathematics, 1(1):67-71, 1990

[22] Edmund Woronowicz. Many argument relations. Formalized Mathematics, 1(4):733-737, 1990.

[23] Edmund Woronowicz. Relations and their basic properties. Formalized Mathematics, 1(1):73-83, 1990.

[24] Edmund Woronowicz. Relations defined on sets. Formalized Mathematics, 1(1):181-186, 1990.

[25] Bo Żhang and Yatsuka Nakamura. The definition of finite sequences and matrices of probability, and addition of matrices of real elements. Formalized Mathematics, 14(3):101108, 2006, doi:10.2478/v10037-006-0012-1.

Received July 4, 2012 